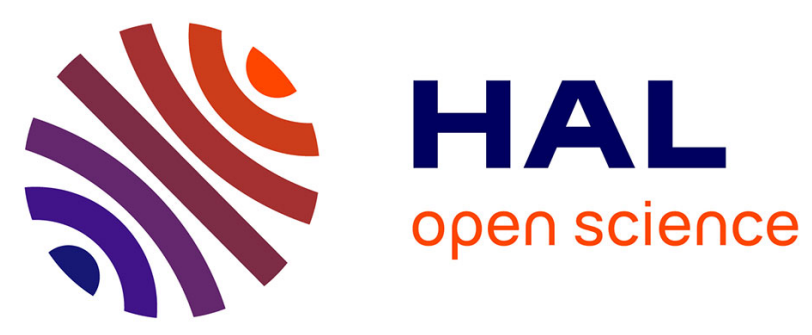

\title{
Risk behaviors in volunteer blood donors who seroconverted for HIV, Abidjan, Côte d'Ivoire 1997 to 2005.
}

Albert Minga, Lambert Dohoun, Yao Abo, Ali Coulibaly, Seidou Konaté, Hervé-Penan Ouattara, Bath K. N'Guessan, François Dabis, Roger Salamon, Charlotte Lewden

\section{To cite this version:}

Albert Minga, Lambert Dohoun, Yao Abo, Ali Coulibaly, Seidou Konaté, et al.. Risk behaviors in volunteer blood donors who seroconverted for HIV, Abidjan, Côte d'Ivoire 1997 to 2005.: HIV infection risk factors in seroconverted blood donors in Abidjan. Transfusion, 2010, 50 (4), pp.888-93. 10.1111/j.1537-2995.2009.02499.x . inserm-00418772

\section{HAL Id: inserm-00418772 https://www.hal.inserm.fr/inserm-00418772}

Submitted on 22 Nov 2010

HAL is a multi-disciplinary open access archive for the deposit and dissemination of scientific research documents, whether they are published or not. The documents may come from teaching and research institutions in France or abroad, or from public or private research centers.
L'archive ouverte pluridisciplinaire $\mathbf{H A L}$, est destinée au dépôt et à la diffusion de documents scientifiques de niveau recherche, publiés ou non, émanant des établissements d'enseignement et de recherche français ou étrangers, des laboratoires publics ou privés. 


\title{
Risks behaviors in volunteer blood donors who seroconverted for HIV, Abidjan, Côte d'Ivoire 1997-2005
}

\author{
ANRS 1220 Primo-CI Study Group. \\ Author affiliation: \\ 1 Programme PAC-CI, Abidjan, Côte d'Ivoire \\ 2 Centre National de Transfusion Sanguine, Abidjan, Côte d'Ivoire \\ 3 Université Victor Segalen Bordeaux 2, ISPED, Bordeaux, France \\ 4 INSERM, U897, Bordeaux, France \\ Corresponding author:
}

Albert Minga' ${ }^{1}$ Lambert Dohoun ${ }^{1}$, Yao Abo ${ }^{1}$, Ali Coulibaly¹, Seidou Konaté ${ }^{2}$, Hervé Penan

Ouattara $^{2}$, Bath K. N'Guessan², François Dabis ${ }^{3,4}$, Roger Salamon ${ }^{3,4}$, Charlotte Lewden ${ }^{3,4}$, for the

Albert Minga, MD, MPH

Programme PAC-CI

18 BP 1954 Abidjan 18, Côte d'Ivoire

Tel: +22507087661 or +22521355278

Fax: +22521355995

e-mail 1 : minga.albert@yahoo.fr

e-mail 2 : albert.minga@pacci.ci

Running head: HIV infection risk factors in seroconverted blood donors in Abidjan 
Abstract: 254 words

Background. The residual risk of HIV transmission from blood products in the Abidjan National Blood Transfusion Centre was estimated to be one in 5780 blood donations over the period 20022004.

Objective: We aimed at describing risk behaviors in blood donors who seroconverted for HIV in Abidjan in order to improve the pre-blood donation selection.

Methods: We investigated the behavioral profile of HIV seroconverters assessed before their HIV diagnosis, during the blood donation selection at the blood bank of Abidjan, and compared it to the profile documented after this HIV diagnosis, at enrollment in the PRIMO-CI cohort. Since 1997, enrollment in this cohort is offered to every blood donor whose delay since HIV seroconversion is $\leq 36$ months.

Results: Among the 418 blood donors who seroconverted for HIV between 1997 and 2005, 241 were enrolled in the cohort. Median age was 28 years and $63 \%$ were men. The median time between the last HIV-negative test and the first positive test was 7 months. Since the last blood donation, $29 \%$ of donors reported unprotected sexual intercourse with multiple casual sexual partners, 55\% unprotected sexual intercourse with one casual sexual partner and 36\% sharing of nail clippers. During the pre-blood donation questionnaire, $69 \%$ of HIV seroconverters had reported unprotected sexual intercourse since the last blood donation (versus 89\% reported postdonation), and 7\% had had multiple casual sexual partners (versus 32\%).

Conclusion: Volunteer blood donors who seroconverted for HIV in Abidjan reported a high proportion of unprotected sexual intercourse with casual sexual partners.

Key words: HIV infection; sexual behavior; sub-Saharan Africa; seroconverters; blood donor 


\section{Introduction}

By the end of 2007, the World Health Organization (WHO) and the United Nations joint Program on AIDS (UNAIDS) estimated that 22.5 million adults (68\% of the people infected worldwide) were living with the human immunodeficiency virus (HIV) in Sub-Saharan Africa (1), and 1.7 million new infections occurred in the region that year.

In Côte d'Ivoire, West Africa, the prevalence of HIV-1 infection in the general population was estimated at $4.7 \%$ in 2005 (2). In this country, most adults are infected through heterosexual transmission and yet data on risk behaviors for HIV transmission are scarce, apart from studies conducted among clients of female sex workers (3). A study conducted in South Africa reported a high HIV prevalence among heterosexual men with multiple sex partners (4). High risk behaviors in blood donors was previously observed in Abidjan (5). In Zimbabwe (6), the rate of heterosexual partner change was high in rural population with high HIV prevalence. A study recently showed the persistence of risk behaviors in the Ugandan rural population (7). In Mali, the HIV prevalence in donations from occasional blood donors was $>14 \%$ \{ Tounkara, 2009 \#727. One of the main obstacles to preventing the sexual transmission of HIV in Africa could be the low percentage of individuals aware of their HIV status, partly due to refusal of performing the HIV test.

In this context where most first time blood donors are unaware of their HIV status, the residual risk of HIV transmission from blood products in the Abidjan National Blood Transfusion Centre was estimated to be one in 5780 blood donations in the period 2002-2004 \{Ouattara, 2006 \#134\}. The prevention of HIV transmission through blood products includes the limitation of transfusions to strictly indicated cases, the fact that blood donors are volunteer, selected and educated and that blood is systematically screened for HIV antibodies (8). Donor selection uses structured questionnaires to exclude recently infected donors at high risk of HIV transmission, 
i.e. donors who may be in the window period of infection before the apparition of HIV antibodies or whose recent HIV infection may not have been detected by the standard serologic screening techniques used at the Blood transfusion centre. This step is particularly crucial in a context of high prevalence of HIV infection like in Cote d'Ivoire. Despite these precautions, blood donors are still detected HIV-positive each year in this blood bank. In industrialized countries, blood donors at high-risk for HIV transmission who were informed on viral transmission and provided free HIV testing self-exclude from donation. Such a practice should be promoted among those in charge of organizing blood donation in countries with limited resources (9). A survey performed in 2001-2002 showed that a majority of the HIV negative regular blood donors in the Abidjan National Blood Transfusion Centre reported multiple partners and an intermittent use of condoms (5). In this study, we aimed at describing risk behaviors in blood donors who seroconverted for HIV in order to improve the pre-blood donation selection process in Abidjan.

\section{Methods}

\section{Patients}

The National Blood Transfusion Centre of Abidjan is coordinating all blood donations in Côte d'Ivoire. Blood donors are non-remunerated adult volunteers. The number of blood donations per year is limited to five in men and four in women. Informed consent for HIV testing, syphilis, hepatitis B and C diagnosis is obtained at each donation. The Blood Bank Medical Centre, the HIV care clinic of the National Blood Transfusion Centre, is in charge of the medical follow-up of individuals diagnosed for one of the screened infections. Patients are informed that the results of these tests will be available at the Blood Bank Medical Centre on a voluntary basis and after an individual post-test counseling. Before each blood donation, a five-minute standard questionnaire is administered individually by a physician. The objective of this questionnaire is to 
exclude donors at risk of HIV, viral hepatitis infection or sexually transmitted infections. Regarding the risk of HIV transmission the exclusion criteria for blood donation are: partner known to be HIV-positive; man who had sex with another man in the previous 3 months or since the previous blood donation; unprotected sexual intercourse with multiple casual sexual partners, sexual intercourse with a female sexual worker or sexually transmitted infection since the previous blood donation.

The French Agency for Research on AIDS (ANRS) 1220 Primo-CI prospective cohort was initiated in June 1997 in the Blood Bank Medical Centre, the HIV care clinic of the National Centre of Blood Transfusion, in Abidjan and aimed to describe the evolution of HIV infection since seroconversion $(10,11)$. HIV-1 seroconverters at the Blood Bank Medical Centre were identified among the repeat blood donors, i.e. individuals who had given blood at least two times. They were followed as early as possible after seroconversion was demonstrated. Enrollment in the Primo-CI cohort was proposed to every blood donor: (i) diagnosed with HIV-1, HIV-2 or HIV-1+2 infection after a blood donation; (ii) HIV seronegative at the preceding donation; (iii) who returned to the clinic to be informed of the HIV-test result and (iv) had an estimated date of seroconversion $<36$ months prior enrollment. The date of seroconversion was estimated as the mid-point between the last negative and the first positive HIV-test. At entry in the ANRS 1220 cohort, participants were administered a questionnaire. The questionnaire administered at enrollment in the Primo-CI cohort was more detailed and response time was longer than for the pre-blood donation questionnaire. All patients with an estimated date of seroconversion between June 1997 and April 2005 were eligible for this analysis. The study protocol was approved by the ethics committee of the national Ivorian program on AIDS and the institutional review board of the ANRS.

\section{Data collection}


We investigated the HIV seroconverters' behavioral profile as documented by the blood donation selection questionnaire administered before HIV diagnosis and compared it to the profile reported within the post-donation questionnaire administered after the HIV diagnosis at enrollment in the PRIMO-CI cohort. The following behavioral data were systematically collected within the questionnaire administered before the blood donation: use of condoms, sexual intercourse with a female sexual worker, change of sexual partners since the last blood donation, sexually transmitted infection, blood transfusion and injection drug use. After informed consent was obtained for enrollment in the cohort, a questionnaire was administered was conducted to document risk factors of HIV infection between the last HIV-negative test and the first HIVpositive test. The following risk behaviors or situations were documented: known HIV-positive sexual partner, unprotected sexual intercourse with multiple casual sexual partners, unprotected sexual intercourse with one casual sexual partner, sexually-transmitted infection, blood transfusion, intravenous drug use and sharing of nail clippers. The duration of the administration of the questionnaire was approximately 30 minutes. The sexual partners of the study participants who agreed to be tested for HIV were tested free of charge.

\section{Biological analysis}

The diagnosis of HIV infection was based on two repeated reactive Enzyme-linked Immunosorbent Assays (Murex ICE 1-0-2®, Abbott, North Chicago, IL, USA; Vironostika HIV Uniform II ${ }^{\circledR}$, Organon Technika BV, Asko Nobel, Boxtel, the Netherlands). Before enrollment in the cohort, HIV infection was confirmed and HIV-1 and HIV-2 typing was established on a subtype blood sample using two ELISA assays (Murex ICE 1-0-2®; Peptilav-Lav 1-2, Pasteur

Diagnostics Marne-la-Coquette, France).

\section{Statistical analysis}


Use of condom, number of casual sexual partners, previous sexual transmitted infection and sexual intercourse with a female sexual worker were compared between two questionnaires: the pre-donation questionnaire administered at the last blood donation and the post-donation questionnaire administered at enrollment in the cohort. Additional risk behaviors were described from the post-donation questionnaire in order to identify the most relevant question that would improve the selection of blood donors. For the present analysis, data were taken into account until April, 2005, when this pre-donation questionnaire was revised by the National Blood Transfusion Centre of Abidjan The paired McNemar's test was used for comparing common qualitative preand post-donation variables. The STATA 9.2 software (Stata Corp LP, College Station, TX, USA) was used for statistical analysis.

\section{Results}

From June 1997 to April 2005, 418 blood donors seroconverted for HIV. Among them, 241 (58\%) were enrolled in the ANRS 1220 PRIMO-CI cohort (HIV-1, $\mathrm{n}=240$ and one HIV-1+2). The reasons for non enrollment in 177 individuals were refusal of post-test counseling $(n=56)$, refusal of enrollment after the confirmation of the HIV test $(n=63)$ and non eligibility $(n=58)$. The 241 adults enrolled were primarily men (63\%), median age was 28 years [interquartile range (IQR) 25-34], 73\% had a post-secondary school level and half were unemployed (table 1). The median time between the last HIV negative test and the first HIV-positive test was seven months, [IQR: 3-18]. The median time from the estimated date of seroconversion to enrollment in the cohort was nine months [IQR: 5-18]. Among the 241 participants, one in three were single, twothirds reported living in stable couple and two men reported having sex with men. Since the last blood donation, the donation that indicated HIV seroconversion, 29\% of the participants reported 
unprotected sexual intercourse with multiple casual sexual partners, 55\% unprotected sexual intercourse with one casual sexual partner and $36 \%$ sharing of nail clippers.

During the pre-blood donation questionnaire, $69 \%$ of HIV seroconverters reported unprotected sexual intercourse since the last blood donation (versus $89 \%$ reported at post-donation), $7 \%$ reported multiple casual sexual partners (versus $32 \%$ ) and only $1 \%$ of these patients reported a sexually transmitted infection (versus 14\%)(Table 2). No significant differences were observed between responses of men and women.

\section{Discussion}

The proportion of unprotected sexual intercourse with casual partners was high in volunteer blood donors who seroconverted for HIV in Abidjan. We observed an underreporting of sexual risk behaviors during the pre-blood donation selection as compared with the post-donation questionnaire. Moreover, risk behaviors of HIV contamination were identified after donation that had not been identified though the pre-blood donation questionnaire.

\section{Limitations of the study}

We compared information collected through two questionnaires that had not been administered at the same time. The 58 patients were not eligible because they did not meet the eligible criteria that were: 18 or more years old; HIV-1 or HIV-1+HIV-2 infected; with a previous HIV negative test, with a positive HIV serology confirmed in two different laboratories; no previous antiretroviral treatment; and with less than 36 months since the date of seroconversion, as estimated by the median date between negative and positive serologies. Moreover, patients living outside from Abidjan and its large surroundings were not enrolled. Changes in risk behaviors may theoretically have occurred between both questionnaires. However, the questionnaire 
administered after HIV diagnosis was the only one available to verify the pre-donation information. Moreover, knowledge of their HIV status may have led individuals to report more easily risk factors than before blood donation. Finally, the Primo-CI enrollment questionnaire collected behaviors between last negative and first positive HIV test and this may have lead to some recall bias. Nevertheless, some questions were identical in both questionnaires and could be compared. Moreover, given the extent of the differences observed, we believe that these findings can be taken into account in order to propose specific improvements in the selection process of blood donors in Abidjan and in blood safety.

\section{Risks behaviors in blood donors compared to other settings}

Despite some improvements in the selection of blood donors since 1999, behaviors at risk of HIV transmission are still largely undetected in regular blood donors. This report from Abidjan shows clearly that some HIV-1 seroconverters under-reported their sexual behavior during the interview before blood donation. The questionnaire administered at enrollment in the Primo-CI cohort was more detailed and duration for interviewing the patient was longer than for the pre-blood donation questionnaire. Moreover, information about HIV status may have encouraged HIV seroconverters to disclose their actual sexual behavior during the interview at enrollment in the Primo-CI cohort.

Several behaviors are known to be associated with the risk of HIV transmission: unprotected sexual intercourse with casual sexual partners, multiple partnerships, unprotected sexual intercourse with multiple casual sexual partners, sexual intercourse with a sex worker, history of sexually transmitted infections and use of shared material (12)(13)(14). Moreover, it is likely that the blood donors recruited within this study were infected by their regular/stable sexual partner. Indeed, several studies in Africa have shown the impact of heterosexual intercourse on the HIV 
incidence in the general population (15)(16)(17)(18). Regarding sexual transmitted infections, a study in Tanzania showed an association between some sexual transmitted diseases such as Herpes simplex virus type-2 and HIV incidence (19).

\section{Risk of HIV transmission though transfusion}

The HIV residual risk between 2002 and 2004 was about one in 5783 blood donations in Abidjan (20). It was 1/8562 in Conakry (21) and 2.6/100 000 donations in South Africa (22). HIV transmission risk due to blood transfusion remains important in Africa (5, 20-23). In industrialized countries this risk is very low (1/2500 000 in France and 1/2 300000 in the USA) $(24,25)$. This may result from a better biological detection of HIV infection, since the HIV RNA is performed in blood donation centers. Moreover, studies performed in industrialized countries showed a self-exclusion of high-risk blood donors for HIV transmission following an enhancement of their knowledge about viral transmission and practice of free testing (9). Such experiences should be encouraging for those in charge of organizing blood donation in countries with limited resources. The fact that blood donors in Abidjan are volunteers (10) may limit the risk of blood donation motivated by earning money. Nevertheless, because nucleic acid testing is not performed in this centre, the window of time before detection of antibodies remains large.

\section{Suggestions for improving the pre-donation selection}

The National Blood Transfusion Centre of Abidjan still detects new seroconversions each year despite prevention interventions at each blood donation, including extensive information on HIV/AIDS and individual counseling (5). The reason why seroconverters who declared their risk behavior during the pre-blood donation questionnaire were allowed to donate should be more deeply investigated. Although we acknowledge the efforts made by the blood centre to exclude blood donors at risk of HIV transmission, repeated blood donors with unknown HIV-serostatus 
sexual partner are still subject to an HIV transmission risk. In this study, some of patients declared having had unprotected sexual intercourse since the last HI- negative test. The HIV status of these partners was not available at Primo-CI study entry. These blood donors are likely to have been infected by their usual sexual partners, and thus additional interventions targeted at stable partnerships are needed in order to reduce the incidence of HIV infection in populations of blood donors. Our study may assist physicians with their decision-making during the predonation selection process. The short time duration of the current pre-selection questionnaire may lead to decisions made too rapidly. The selection method preceding blood donation should be improved. Specific interventions targeted toward stable partnerships are needed and the sexual partners of regular blood donors should be tested for HIV. Notification by blood donors of all sexual transmitted diseases and all behaviors at risk of HIV transmission has to be encouraged. Moreover, the interview, including individual support, must be systematically repeated before each donation in order to exclude high-risk blood donors.

In conclusion, including extensive information on HIV/AIDS and individual counseling is crucial for selecting blood donors. In the long term, we believe that educating blood donors will remain one of the keys to guaranteeing blood safety. It will be important to further link such an educational initiative with the broader national HIV/AIDS program and to promote blood donation as part of a safe lifestyle to prevent the spread of HIV through blood transfusion and high-risk behaviors. The blood donors at risk of HIV transmission should exclude themselves and be discouraged to continue giving blood through adequate counseling. The information provided should clearly state that safe behaviors and self-exclusion for blood donation can prevent HIV transmission by blood transfusion.

\section{ACKNOWLEDGMENTS}


The authors thank Drs Koffi Nicole Abé and Hyda Jules of the National Blood Transfusion

Centre for contributing to this study.

\section{References:}

1. UNAIDS, World Health Organization. AIDS epidemic update. 2006 [cited 2007/06/29]. Available from: http://www.who.int/hiv/mediacentre/2006_EpiUpdate_en.pdf

2. UNAIDS, World Health Organization. AIDS epidemic update. 2006 [cited 2007/06/29]. Available from: http://www.who.int/hiv/mediacentre/2006_EpiUpdate_en.pdf

3. Vuylsteke B, Ghys P, Traoré M, Konan Y, Mah-Bi G, Maurice C, et al. HIV prevalence and risk behavior among clients of female sex workers in Abidjan, Côte d'Ivoire. AIDS 2003;17:1691-4.

4. Chopra M, Townsend L, Johnston L, Mathews C, Tomlinson M, O'bra H, et al. Estimating HIV prevalence and risk behaviors among high-risk heterosexual men with multiple sex partners: use of respondent-driven sampling. J Acquir Immune Defic Syndr 2009;51:72-7.

5. Minga A, Huët C, Dohoun L, Abo Y, Bonard D, Gourvellec G, et al. Behavior assessment of blood donors facing the risk of HIV infection, Abidjan, Cotê D'Ivoire, 2001-2002. J Acquir Immune Defic Syndr 2005;38:618-21.

6. Lopman B, Nyamukapa C, Hallett T, Mushati P, Spark-du Preez N, Kurwa F, et al. Role of widows in the heterosexual transmission of HIV in Manicaland, Zimbabwe, 19982003. Sex Transm Infect 2009;85 Suppl 1:i41-8.

7. Biraro S, Shafer L, Kleinschmidt I, Wolff B, Karabalinde A, Nalwoga A, et al. Is sexual risk taking behaviour changing in rural south-west Uganda? Behaviour trends in a rural population cohort 1993-2006. Sex Transm Infect 2009;85 Suppl 1:i3-11.

8. King S, AuBuchon J, Barrowman N, Follea G, Giroux M, Kim W, et al. Consensus statement from the consensus conference on blood-borne human immunodeficiency virus and hepatitis: optimizing the donor-selection process. Vox Sanguinis 2002;83:188-193.

9. Sharma U, Schreiber G, Glynn S, Nass C, Higgins M, Tu Y, et al. Knowledge of HIV/AIDS transmission and screening in United States blood donors. Transfusion 2001;41:1341-50.

10. Salamon R, Marimoutou C, Ekra D, Minga A, Nerrienet E, Huet C, et al. Clinical and biological evolution of HIV-1 seroconverters in Abidjan, Cote d'Ivoire, 1997-2000. J Acquir Immune Defic Syndr 2002;29:149-157. 
11. Minga A, Huet C, Coulibaly I, Abo Y, Dohoun L, Bonard D, et al. [Profile of HIV infected patients among blood donors in Abidjan, Cote d'Ivoire (1992-1999)].

Bull Soc Pathol Exot 2005;98:123-6.

12. Ryder R, Kamenga C, Jingu M, Mbuyi N, Mbu L, Behets F. Pregnancy and HIV-1 incidence in 178 married couples with discordant HIV-1 serostatus: additional experience at an HIV-1 counselling centre in the Democratic Republic of the Congo.

Trop Med Int Health 2000;5:482-7.

13. Quigley M, Morgan D, Malamba S, Mayanja B, Okongo M, Carpenter L, et al. Casecontrol study of risk factors for incident HIV infection in rural Uganda.

J Acquir Immune Defic Syndr 2000;23:418-25.

14. Hugonnet S, Mosha F, Todd J, Mugeye K, Klokke A, Ndeki L, et al. Incidence of HIV infection in stable sexual partnerships: a retrospective cohort study of 1802 couples in Mwanza Region, Tanzania. J Acquir Immune Defic Syndr 2002;30:73-80.

15. Schmid G, Buve A, Mugyenyi P, Garnett G, Hayes R, Williams B, et al. Transmission of HIV-1 infection in sub-Saharan Africa and effect of elimination of unsafe injections. Lancet 2004;363:482-8.

16. Okware S, Opio A, Musinguzi J, Waibale P. Fighting HIV/AIDS: is success possible? Bull World Health Organ 2001;79:1113-20.

17. Ali M, Cleland J, Shah I. Condom use within marriage: a neglected HIV intervention. Bull World Health Organ 2004;82:180-6.

18. Todd J, Grosskurth H, Changalucha J, Obasi A, Mosha F, Balira R, et al. Risk factors influencing HIV infection incidence in a rural African population: a nested case-control study. J Infect Dis 2006;193:458-66.

19. del Mar Pujades Rodriguez M, Obasi A, Mosha F, Todd J, Brown D, Changalucha J, et al. Herpes simplex virus type 2 infection increases HIV incidence: a prospective study in rural Tanzania. AIDS 2002;16:451-62.

20. Ouattara H, Siransy-Bogui L, Fretz C, Diane K, Konate S, Koidio A, et al. Residual risk of HIV, HVB and HCV transmission by blood transfusion between 2002 and 2004 at the Abidjan National Blood Transfusion Center. Transfus Clin Biol 2006;13:212-5.

21. Loua A, Sow E, Magassouba F, Camara M, Balde M. Evaluation of residual infectious risk among blood donors in National Center of Blood Transfusion in Conakry.

Transfus Clin Biol 2004;11:98-100. 
22. Heyns Adu P, Benjamin R, Swanevelder J, Laycock M, Pappalardo B, Crookes R, et al. Prevalence of HIV-1 in blood donations following implementation of a structured blood safety policy in South Africa. JAMA 2006;295:519-26.

23. Tounkara A, Diakite M, Noumsi G, Sarro Y, Siddiqui S, Parta M. Retrospective surveillance of HIV prevalence in blood donors can help in the selection of the best social group for blood donation in Mali. Transfus Med 2009;19:252-9.

24. Pillonel J, Laperche $\mathrm{S}$. [Trends in residual risk of transfusion-transmitted viral infections (HIV, HCV, HBV) in France between 1992 and 2002 and impact of viral genome screening (Nucleic Acid Testing)]. Transfus Clin Biol 2004;11:81-6.

25. Busch M, Glynn S, Stramer S, Strong D, Caglioti S, Wright D, et al. A new strategy for estimating risks of transfusion-transmitted viral infections based on rates of detection of recently infected donors. Transfusion 2005;45:254-64. 